УДК 330.322 .5

\title{
Ю. Ю. Стецук
}

Российский экономический университет им. Г.В. Плеханова, Москва, e-mail: stecuk.yu@edu.rea.ru

\section{А. А. Струкова}

Российский экономический университет им. Г.В. Плеханова, Москва, e-mail: anastasiia.strukova2003@mail.ru

\section{ПОСТАНОВКА ЗАДАЧИ ДИНАМИЧЕСКОЙ ОПТИМИЗАЦИИ ПРОИЗВОДСТВЕННОЙ СФЕРЫ ПРЕДПРИЯТИЯ ПО РЫНОЧНОМУ КРИТЕРИЮ}

Ключевые слова: производственная сфера предприятия, рабочий капитал, модель «затратывыпуск», неоклассическая производственная функция, динамическая модель производственной сферы предприятия, критерии оптимальности, риск структуры капитала, управляемые и неуправляемые параметры, аналитические методы, методы оптимизации, задачи нелинейного целочисленного программирования.

Выбор оптимального по экономическому критерию варианта организации денежных и материальных потоков производственной сферы предприятия - актуальная с позиции повышения его эффективности и конкурентоспособности по затратам задача, решение которой включается в процесс разработки рыночной и внутрифирменной стратегий. До настоящего времени эта задача широко не освящена в экономической литературе: отсутствуют обоснованные постановки задач оптимизации, не в полной мере разработаны оптимизационные модели и не представлены практические расчеты оптимальных вариантов. В настоящей публикации авторы рассматривают эти задачи в приложении к предприятию, функция «затраты - выпуск» которого корректно описывается неоклассической производственной функцией. Приводятся соответствующие этому варианту постановка задачи, собственно динамическая модель, перечень критериев оптимальности и рисковых ограничений, результаты расчетов по динамической модели для различных комбинаций экзогенных и эндогенных параметров. В частности, показано, что степень однородности производственной функции (масштаб производства) оказывает определяющее влияние на динамику выпуска, увеличивая затраты в случае планирования высоких объемов производства. Среди управляемых параметров модели важную роль играет темп инвестиций в рабочий капитал предприятия из собственных источников, повышая/снижая выпуск. Ограничение на рискструктуры рабочего капитала оказывает определяющее влияние на динамику результата в производственной сфере: с ростом норматива финансовой зависимости выпуск растет быстрее риска банкротства по причине высокой отдачи рычага капитала.

\section{Yu. Yu. Stetsuk}

Plekhanov Russian University of Economics, Moscow, e-mail: stecuk.yu@edu.rea.ru

\section{A. A. Strukova}

Plekhanov Russian University of Economics, Moscow, e-mail: anastasiia.strukova2003@mail.ru

\section{FORMULATION OF THE PROBLEM OF DYNAMIC OPTIMIZATION OF THE PRODUCTION SPHERE OF THE ENTERPRISE BY MARKET CRITERION}

Keywords: production sphere of an enterprise, working capital, input-output model, neoclassical production function, dynamic model of production sphere of an enterprise, optimality criteria, capital structure risk, controlled and uncontrolled parameters, analytical methods, optimization methods, nonlinear integer programming problems.

The choice of the optimal option for the economic criterion of the organization of monetary and material flows of the production sphere of the enterprise is an actual problem from the position of increasing its efficiency and cost competitiveness, the solution of which is included in the process of developing market and intra-company strategies. However, this task has not been widely discussed in the economic literature: optimization models are not fully developed, practical calculations of the optimal variants are not presented. In this publication the authors consider these problems in application to an enterprise, the function "inputoutput" of which is correctly described by the neoclassical production function, presenting the problem statement corresponding to this variant, the dynamic model, list of optimality criteria and risk restrictions, results of calculations by the dynamic model for various combinations of exogenous and endogenous parameters. In particular, it is shown that the degree of homogeneity of the production function has a decisive influence 
on the dynamics of output, increasing costs in the case of planning high production volumes. Among the controllable parameters of the model an important role is played by the rate of investment in the working capital of the enterprise from its own sources, increasing/decreasing the output. The restriction on the risk of the working capital structure has a determining influence on the dynamics of the result in the production sphere: as the financial dependence norm increases, output grows faster than the risk of bankruptcy due to the high return on capital leverage.

\section{Введение}

Эта публикация является прямым продолжением исследований профессора М.А. Халикова и его учеников по проблематике динамической оптимизации производственной сферы предприятия с критериями эффективности и рентабельности затрат, финансируемых из собственных и заемных источников и с ограничениями, включающими и ограничение на риск структуры пассивов рабочего капитала. В первую очередь, здесь следует процитировать работы указанного коллектива авторов [1,3-6,11], отметив, что предлагаемая в статье постановка задачи динамической оптимизации является оригинальной в плане выбранного объекта приложения - рассматривается вариант предприятия, зависимость в паре «затраты-выпуск» которого корректно описывается неоклассической производственной функцией [9].

\section{Материалы и методы исследования}

Методологическую основу исследования составили работы отечественных и зарубежных учёных по:

1) проблемам моделирования производственной сферы предприятия в условиях неопределенности и риска [12,17];

2) оптимизации социально-экономических систем на микроуровне [2,8];

3) методам дискретной оптимизации в непрерывной и дискретной постановках [14-16];

4) методам оценки и учета в моделях производственной сферы предприятия внешних и внутренних рисков $[13,15]$.

Цель исследования - продолжение исследований, начатых в публикациях [3-5] и связанных с разработкой экономико-математического инструментария моделей и методов выбора оптимальных по экономическому критерию вариантов управления денежными и материальными потоками производственной сферы предприятия в части разработки и адаптации постановок задач и алгоритмов их динамической оптимизации на выбранном плановом горизонте.

\section{Результаты исследования и их обсуждение}

1. Производственная сфера предприятия и метод динамического моделирования ее денежных потоков.

Объектом исследований в этой статье является производственная сфера рыночной деятельности корпорации (предприятия частной формы собственности), в рамках которой осуществляются планирование и организация основного производственного процесса, снабжение, подготовка производства и сбыт (реализация) готовой продукции.

Целью производственной деятельности является получение эффекта в виде прибыли или другого дохода от активов производственной сферы, используемых в технологическом процессе.

Активы производственной сферы предприятия (в совокупности образуют рабочий капитал) по способу списания на затраты производственной деятельности подразделяются на постоянные и переменные.

Пассивы рабочего капитала формируются из собственных средств, производственного сегмента предприятия и заемных, привлекаемых из внешних источников (на платной основе) на покрытие прямых и косвенных производственных затрат.

Основой моделирования производственного сегмента предприятия является производственная функция, в аналитической или табличной форме связывающая выпуск продукции в натуральном или стоимостном выражении (стоимость «на выходе») с затратами активов рабочего капитала, используемых в технологическом процессе ее производства (стоимость «на входе»). В частности, в работе используется аналитическое выражение для неоклассической производственной функции, а также основные ее свойства, связанные с интерпретацией коэффициента однородности (эти данные заимствованы из работ Б.Г. Клейнера и М.А. Халикова) $[1,9]$.

Приведем основные соотношения динамической модели производственной сферы предприятия, связывающие активы и пассивы, включаемые в рабочий капитал на последовательных временных интерва- 
лах $\mathrm{t}-1$ (предыдущий) и $\mathrm{t}$ (текущий), где $\mathrm{t} \in 0, \mathrm{~T}$ ( $\mathrm{T}-$ горизонт планирования):

1) $\mathrm{PK}_{\mathrm{t}}=\overline{\mathrm{PK}}_{\mathrm{t}-1}$,

т.е рабочий капитал производственной сферы на начало текущего временного интервала совпадает по величине и составляющим с рабочим капиталом на конец предыдущего временного интервала;

2) $\overline{\mathrm{PK}}_{\mathrm{t}}=(1-\sigma) \times \mathrm{PK}_{\mathrm{t}}+\gamma_{\mathrm{t}} \times \overline{\mathrm{y}}_{\mathrm{t}}$,

рабочий капитал (в части активов) на конец временного интервала t образуется из рабочего капитала на начало этого временного интервала (за исключением амортизируемой доли) и собственных инвестиций предприятия, и валовой прибыли $\overline{\mathrm{y}}_{\mathrm{t}}$ периода $\mathrm{t}$ $\left(\gamma_{t}\right.$ - доля собственных инвестиций в рабочий капитал для периода $\mathrm{t})$;

3) $\bar{y}_{t}=(1-\tau)\left(p_{t} \times y_{t}-w_{t}-\left(1+\rho_{t}\right) \times 3 K_{t}\right)$,

где $\tau$ - налог на прибыль; $\mathrm{p}_{\mathrm{t}}$ - цена реализации произведенной в периоде $\mathrm{t}$ продукции объемом $y_{t} ; w_{t}$ - нормируемые постоянные и переменные затраты на выпуск продукции объемом $y_{t} ; \rho_{t}-$ ставка краткосрочного кредита объемом $3 \mathrm{~K}_{t}$ в пассивах рабочего капитала производственной сферы предприятия для периода $\mathrm{t}$.

Объем $y_{t}$ производства для периода $t$ не может превышать прогнозируемого спроca $\mathrm{SP}_{\mathrm{t}}$ на продукцию предприятия для этого периода и его производственной мощности, ограниченной постоянными и переменными активами рабочего капитала $\mathrm{PK}_{t}$ на начало временного интервала t. Напомним, что для предприятия с неоклассической производственной функцией справедливо:

$$
\mathrm{y}_{\mathrm{t}} \leq\left(\frac{\mathrm{PK}_{\mathrm{t}}}{\mathrm{c}_{\mathrm{t}}(1)}\right)^{\alpha},
$$

где $\mathrm{c}_{\mathrm{t}}(1)$ - удельные затраты на производство продукции для временного интервала $\mathrm{t}$; $\alpha$ - степень однородности производственной функции предприятия.

Таким образом, для $y_{t}$ справедливо:

4) $\mathrm{y}_{\mathrm{t}}=\min \left\{\mathrm{SP}_{\mathrm{t}} ;\left(\frac{\mathrm{PK}_{\mathrm{t}}}{\mathrm{c}_{\mathrm{t}}(1)}\right)^{\alpha}\right\}$

$$
\mathrm{w}_{\mathrm{t}}=c_{\mathrm{t}}(1) \times \mathrm{y}_{\mathrm{t}}^{1 / \alpha}
$$

где $\mathrm{w}_{\mathrm{t}}$ - отмеченные в соотношении (3) полные затраты производственной сферы предприятия для временного интервала t;
5) $3 \mathrm{~K}_{\mathrm{t}}=\mathrm{PK}_{\mathrm{t}} \times\left(1-\mathrm{k}_{\mathrm{t}}\right)$,

где $\mathrm{k}_{\mathrm{t}}$ - коэффициент автономии структуры пассивов рабочего капитала предприятия, выбранный для временного интервала t (отношение собственного капитала $\mathrm{PK}_{\mathrm{t}}$ производственной сферы к полному капиталу $\left.\mathrm{PK}_{t}+3 \mathrm{~K}_{t}\right)$;

6) $\mathrm{D}_{\mathrm{t}}=\left(1-\gamma_{\mathrm{t}}\right) \times \overline{\mathrm{y}}_{\mathrm{t}}$,

где $\mathrm{D}_{\mathrm{t}}$ - объем средств из валовой прибыли производственной сферы предприятия для временного интервала $t$, выделяемые на непроизводственное потребление (в частности, на выплату дивидендов).

Приведенные соотношения (1) - (8) в совокупности описывают баланс денежных и материальных потоков производственной сферы предприятия на временных интервалах $\mathrm{t}(\mathrm{t}=\overline{1, \mathrm{~T}})$.

В таблице 1 представим описание используемых в этих соотношениях экзогенных (неуправляемых) и эндогенных (управляемых) параметров.

Таким образом, балансы денежных и материальных потоков производственной сферы предприятия для временного интервала $\mathrm{t}$ $(\mathrm{t}=\overline{1, T})$, задаются соотношениями $(2)-(8)$, а переход от одного временного интервала к другому соотношением (1).

Манипулируя параметрами $\mathrm{k}_{\mathrm{t}}$ и $\gamma_{\mathrm{t}}$ (соответственно коэффициент автономии структуры пассива рабочего капитала производственной сферы и доля отчислений из прибыли на инвестиции в рабочий капитал), можно ставить и решать следующие задачи оптимального управления производственным сегментом предприятия:

a) максимально увеличить производственную мощность (объем рабочего капитала) и выпуск продукции к концу планового периода Т) в условиях прогнозируемых интервалов измерения рыночного спроса и цен на продукцию и с учетом выбранных нормативов на риск структуры рабочего капитала (коэффициент автономии к концу планового периода T, а также на промежуточных временных интервалах не должен быть меньше предельного значения);

б) максимально повысить инвестиционную привлекательность производственного сегмента предприятия к концу планового периода Т в условиях прогнозируемых интервалов изменения рыночного спроса и цен на продукцию путем увеличения дивидендных выплат с учетом пороговых значений рентабельности активов рабочего капитала и риска его структуры; 
Экзогенные и эндогенные параметры динамической модели производственной сферы предприятия

\begin{tabular}{|c|c|c|c|}
\hline $\begin{array}{c}\text { Обозначение } \\
\text { параметра }\end{array}$ & Описание параметра & $\begin{array}{c}\text { Обозначение } \\
\text { параметра }\end{array}$ & Описание параметра \\
\hline$\sigma(\sigma>0)$ & $\begin{array}{l}\text { Коэффициент амортизации акти- } \\
\text { вов рабочего капитала предпри- } \\
\text { ятия для временного интервалаt } \\
\text { (неуправляемый параметр) }\end{array}$ & $\mathrm{k}_{\mathrm{t}}, \mathrm{k}_{\mathrm{t}} \in(0 ; 1]$ & $\begin{array}{l}\text { Коэффициент автономии структуры } \\
\text { пассивов рабочего капитала, уста- } \\
\text { навливаемый для временного интер- } \\
\text { вала t (управляемый параметр) }\end{array}$ \\
\hline$\gamma_{t}, \gamma_{t} \in(0 ; \alpha)$ & $\begin{array}{l}\text { Доля отчислений из валовой при- } \\
\text { были, полученной в производ- } \\
\text { ственной сфере предприятия в вре- } \\
\text { менном интервале t, направляемая } \\
\text { в инвестиции в активы рабочего } \\
\text { капитала (управляемый параметр). }\end{array}$ & $\alpha(\alpha>0)$ & $\begin{array}{l}\text { Степень однородности производ- } \\
\text { ственной функции предприятия (не- } \\
\text { управляемый параметр) }\end{array}$ \\
\hline$\tau(\tau>0)$ & $\begin{array}{l}\text { Ставка налога на прибыль } \\
\text { (неуправляемый параметр) }\end{array}$ & $c_{t}(1)$ & $\begin{array}{l}\text { Удельные затраты активов рабоче- } \\
\text { го капитала в расчете на единицу } \\
\text { продукции производственной сфе- } \\
\text { ры для временного интервала t (не- } \\
\text { управляемый параметр) }\end{array}$ \\
\hline $\mathrm{p}_{\mathrm{t}}\left(\mathrm{p}_{\mathrm{t}}>0\right)$ & $\begin{array}{l}\text { Цена на производимую предпри- } \\
\text { ятием продукцию для временного } \\
\text { интервала t (неуправляемый пара- } \\
\text { метр) }\end{array}$ & $\mathrm{SP}_{\mathrm{t}}$ & $\begin{array}{l}\text { Величина рыночного спроса на про- } \\
\text { дукцию предприятия для времен- } \\
\text { ного интервала t (неуправляемый } \\
\text { параметр) }\end{array}$ \\
\hline$\rho_{\mathrm{t}}, \rho_{\mathrm{t}} \in(0 ; \alpha)$ & $\begin{array}{l}\text { Цена краткосрочного кредита для } \\
\text { временного интервала t (неуправ- } \\
\text { ляемый параметр) }\end{array}$ & $\mathrm{PK}_{1}$ & $\begin{array}{l}\text { Величина активов рабочего капита- } \\
\text { ла производственной сферы пред- } \\
\text { приятия на начало первого времен- } \\
\text { ного интервала (неуправляемый } \\
\text { параметр) }\end{array}$ \\
\hline
\end{tabular}

в) максимально повысить рентабельность рабочего капитала производственной сферы предприятия к концу планового периода Т в условиях прогнозируемых интервалов изменения рыночных спроса и цен на продукцию с учетом пороговых значений риска структуры рабочего капитала и доли отчислений из прибыли на непроизводственное потребление.

Перечисленные постановки задач, конечно же, не исчерпывают поле решаемых проблем управления материальными и денежными потоками производственной сферы хозяйствующего в рыночной экономике предприятия. Однако по содержанию и элементному наполнению перечисленные задачи весьма актуальны с практической точки зрения, и возможности эмпирических исследований на основе построенных формализованных моделей.

Рассмотрим эти модели.

- Модель задачи п. а), кроме соотношений (1)-(8), включает ограничения:
- на прогнозируемые интервалы изменения рыночных спроса и цен реализации продукции предприятия:

$$
\mathrm{SP}_{\mathrm{t}} \in\left[\mathrm{S}_{\mathrm{p}} ; \overline{\mathrm{S}}_{\mathrm{p}}\right], \quad \mathrm{p}_{\mathrm{t}} \in[\mathrm{p} ; \overline{\mathrm{p}}],
$$

где в ограничениях указаны соответственно нижние и верхние границы прогнозируемых интервалов изменения спроса и цен;

- на максимальный риск структуры пассивов рабочего капитала:

$$
\mathrm{k}_{\mathrm{t}} \leq \mathrm{k}, \mathrm{t}=\overline{1, \mathrm{~T}}
$$

Критерий задачи п а):

$$
\max \overline{\mathrm{y}}_{\mathrm{t}}
$$

Таким образом, задачи п. а) корректно описывается системой выражений (1)-(11).

- Модель задачи в), кроме соотношений (1)-(8), включает ограничения (9), (10) и ограничение на минимальный уровень рентабельности активов рабочего капитала на всем плановом горизонте $\mathrm{t}=[1 ; \mathrm{T}]$ : 


$$
\frac{2 \overline{y_{t}}}{\mathrm{PK}_{t}+\overline{\mathrm{PK}_{t}}} \geq \text { ren, }
$$

где ren - порог рентабельности средней за временной интервал $t$ величины активов рабочего капитала производственной сферы предприятия.

Критерий задачи - максимум отчислений на непроизводственное потребление к концу периода планового периода Т:

$$
\mathrm{D}_{\mathrm{t}} \rightarrow \max ,
$$

Таким образом, модель задачи в) описывается системой выражений (1)-(10), (12), (13).

- Модель задачи с), кроме соотношений (1)-(8), также включает ограничения (9), (10) и ограничение на нижний порог доли отчислений на непроизводственное потребление:

$$
1-\gamma_{\mathrm{t}} \geq \text { Liv, }
$$

где Liv - нижний порог доли дивидендных выплат из прибыли производственной сферы предприятия.

Критерий этой задачи - максимум рентабельности активов рабочего капитала производственного сегмента предприятия к концу планового периода T:

$$
\frac{2 \mathrm{y}_{\mathrm{T}}}{\mathrm{PK}_{\mathrm{T}}+\overline{\mathrm{PK}_{\mathrm{T}}}} \rightarrow \max
$$

2. Эмпирические расчеты динамики производственной сферы предприятия с критерием (11).

Исследования экономической динамики производственной сферы предприятия, функционирующего на конкурентных товарных, ресурсных и финансовых рынков и для частного случая линейной зависимости в паре «затраты-выпуск» (степень однородности производственной функции $\alpha=1$ ), проводились М.А. Халиковым и его учениками: А.М. Антиколь, Э.А. Бабаян, Р.М. Расуловым и Д.А. Безуховым [1,3-5].

Авторами показано, что в этом случае экономическая динамика предприятия корректно описывается однородными разностными уравнениями второго порядка. Если соответствующее характеристическое уравнение разрешимо в действительных числах, то динамика выпуска описывается возрастающей или убывающей экспоненциальной функцией, если в комплексных, то - периодической волнообразной функцией (растущей или затухающей).
Для исследования экономической динамики предприятия с нелинейной неоклассической производственной функцией, задаваемой уравнениями (1)-(11), предлагается рассмотреть случаи растущей $(\alpha>1)$ и падающей $(\alpha<1)$ отдачи от масштаба производства.

Не ограничивая общности рассуждений, будем считать фиксированными: ставку $\tau$ налогообложения прибыли, цены p - товарного и $\rho-$ финансового рынков, а также технологические константы: с(1) (удельные затраты) и $\mathrm{PK}_{1}$ (рабочий капитал предприятия в начале первого временного интервала - в натуральных единицах).

Практические расчеты экономической динамики производственной сферы предприятия с неоклассической производственной функцией проведем для случаев $\alpha<1$, $\alpha>1$ и различных комбинаций управляемых параметров $\gamma_{\mathrm{t}}$ и $\beta_{\mathrm{t}}$ и для следующих рыночных и технологических констант:

$$
\begin{gathered}
\tau=0,20 ; p=2 ; c(1)=1,2 ; \\
\rho=0,15 ; \mathrm{v}_{1}=16 ; \mathrm{CPK}_{1}=0 .
\end{gathered}
$$

Характер динамики конечного продукта (нераспределенной прибыли) для различных комбинаций экзогенных и эндогенных параметров отражен в таблице 2 .

Расчеты экономической динамики производственной сферы предприятия с неоклассической производственной функцией, основанные на соотношениях (1)-(12), позволили сделать следующие выводы, часть из которых согласуются с выводами работ $[1,3,5]$, полученными для частного случая линейной производственной функции:

1. Масштаб производства (степень однородности производственной функции), являясь фактором модели «выпуск-затраты», существенно влияет на экономическую динамику предприятия, что прямо следует из результатов, приведенных в таблице 2 для случаев растущей и снижающейся отдачи от масштаба производства.

2. Выбор управляемых параметров модели «выпуск-затраты» $\left(\gamma_{\mathrm{t}}-\right.$ доля инвестиций из собственных средств в активы рабочего капитала, $\mathrm{k}_{\mathrm{t}}$ - доля собственных средств в пассивах рабочего капитала) является корректным.

3. Пороговым значением показателя $\gamma_{t}$ является 0,4, что подтверждается сравнительным анализом динамики выпуска для значений показателя соответственно меньших и больших приведенного значения. 
Таблица 2

Варианты расчетов экономической динамики производственной сферы предприятия с неоклассической производственной функцией с критерием (11)

\begin{tabular}{|c|c|c|c|}
\hline$\alpha$ & $\gamma_{t}$ & $\boldsymbol{k}_{t}$ & Динамика «выпуск-затраты» \\
\hline 0,8 & 0,1 & 0,9 & Экспоненциальное падение \\
\hline 0,8 & 0,1 & 0,5 & Экспоненциальное падение \\
\hline 0,8 & 0,1 & 0,1 & Умеренное экспоненциальное падение \\
\hline 0,8 & 0,4 & 0,9 & Экспоненциальное падение \\
\hline 0,8 & 0,4 & 0,5 & Умеренное экспоненциальное падение \\
\hline 0,8 & 0,4 & 0,1 & Квазилинейный рост \\
\hline 0,8 & 0,6 & 0,9 & Умеренное экспоненциальное падение \\
\hline 0,8 & 0,6 & 0,5 & Квазипостоянный выпуск \\
\hline 0,8 & 0,6 & 0,1 & Заметный экспоненциальный рост \\
\hline 0,8 & 0,9 & 0,9 & Квазилинейное падение \\
\hline 0,8 & 0,9 & 0,5 & Линейный рост \\
\hline 0,8 & 0,9 & 0,1 & Экспоненциальный рост \\
\hline 1,2 & 0,1 & 0,9 & Умеренное экспоненциальное падение \\
\hline 1,2 & 0,1 & 0,5 & Умеренное экспоненциальное падение \\
\hline 1,2 & 0,1 & 0,1 & Заметный экспоненциальный рост \\
\hline 1,2 & 0,4 & 0,9 & Квазилинейный рост \\
\hline 1,2 & 0,4 & 0,5 & Экспоненциальный рост \\
\hline 1,2 & 0,4 & 0,1 & Заметный экспоненциальный рост \\
\hline 1,2 & 0,6 & 0,9 & Экспоненциальный рост \\
\hline 1,2 & 0,6 & 0,5 & Заметный экспоненциальный рост \\
\hline 1,2 & 0,6 & 0,1 & Сверхсильный экспоненциальный рост \\
\hline 1,2 & 0,9 & 0,9 & Заметный экспоненциальный рост \\
\hline 1,2 & 0,9 & 0,5 & Заметный экспоненциальный рост \\
\hline 1,2 & 0,9 & 0,1 & Сверхсильный экспоненциальный рост \\
\hline
\end{tabular}

4. Аналогично случаю линейной производственной функции важную роль для улучшения динамики выпуска играет фактор налогового щита: с ростом финансового рычага (доли заемных средств в производственном капитале) выпуск и рентабельность затрат растут.

В целом, приведенные теоретические обоснования модели динамики «выпуск-за- траты» и практические расчеты по модели с критерием на максимум выпуска в конце планового периода убедительно демонстрируют актуальность постановки задачи оптимизации экономической динамики производственного сегмента предприятия в условиях выделения в его структуре центров прибыли и инвестиций.

\section{Библиографический список}

1. Антиколь А.М., Халиков М.А. Нелинейные модели микроэкономики: учеб. пособие. М.: ФГБОУ ВПО «РЭУ им. Г.В. Плеханова», 2011. 156 с.

2. Аоки М. Введение в методы оптимизации. Основы и приложения нелинейного программирования. М.: Наука, 1977. 343 с. 
3. Бабаян Э.А., Расулов Р.М., Халиков М.А. Динамические модели «затраты-выпуск» // Экономика природопользования. 2013. № 2. С. 3-16.

4. Безухов Д.А., Халиков М.А. Выбор оптимального варианта обновления основного капитала предприятия с учетом рисков производственной сферы // Фундаментальные исследования. 2015. № 4. C. 191-198.

5. Безухов Д.А., Халиков М.А. Математические модели и практические расчеты оптимальной структуры производственного капитала предприятия с неоклассической производственной функцией // Фундаментальные исследования. 2014. № 11-1. С. 114-123.

6. Бельченко С.В., Халиков М.А., Щепилов М.В. Управление транзакционными издержками интегрированной группы предприятий: Модели и методы. М.: ЗАО «Гриф и К», 2011. 172 с.

7. Горский М.А. Теоретический подход и численный метод поиска квазиоптимального решения нелинейной дискретной задачи большой размерности // Экономический журнал Высшей школы экономики. 2019. T. 23. № 3. С.465-482.

8. Грибов А.Ф. Нелинейная модель оптимизации операционной деятельности предприятия // Фундаментальные исследования. 2016. № 2-1. С. 140-144.

9. Клейнер Г.Б. Производственные функции: теория, методы, применение. М.: Финансы и статистика, 1986. 239 c.

10. Колемаев В.А. Математические методы и модели исследования операций. М.: ЮНИТИ-ДАНА, 2012. $592 \mathrm{c}$.

11. Maximov D.A., Khalikov M.A. Prospects of institutional approach to production corporation assets assessment // Actual Problems of Economics. 2016. Vol. 183. № 9. P. 16-25.

12. Minniti A., Turino F. Multi-product firms and business cycle dynamics // European Economic Review. 2013. Vol. 57. P. 75-97.

13. Максимов Д.А., Халиков М.А. Методы оценки и стратегии обеспечения экономической безопасности предприятия. М.: ЗАО «Грифи К», 2012. 220 с.

14. Максимов Д.А., Халиков М.А. Моделирование инвестиционной деятельности предприятия, ориентированной на рост производства и снижение производственного риска // Ученые записки Российской Академии предпринимательства. 2008. № 16. С. 70-80.

15. Khalikov M.A., Maximov D.A., Shabalina U.M. Risk Indicators Fnd Risk Management Models for an Integrated Group of Enterprises // Journal of Applied Economic Sciences. 2018. Vol. 13. № 1 (55). P. 52-64.

16. Халиков М.А. Дискретная оптимизация планов повышения надежности функционирования экономических систем // Финансовая математика: сб. ст. М.: МГУ, 2001. С. 281-295.

17. Халиков М.А., Максимов Д.А. Об одном подходе к анализу и оценке ресурсного потенциала предприятия // Международный журнал прикладных и фундаментальных исследований. 2015. № 11-2. C. 296-300. 